\title{
Universal diffusion near the golden chaos border
}

\author{
S.Ruffo ${ }^{(a)}$, D.L.Shepelyansky ${ }^{(b)}$ \\ Laboratoire de Physique Quantique, Université Paul Sabatier, \\ 118, route de Narbonne, 31062 Toulouse, France
}

(November 9, 2018)

\begin{abstract}
We study local diffusion rate $D$ in Chirikov standard map near the critical golden curve. Numerical simulations confirm the predicted exponent $\alpha=5$ for the power law decay of $D$ as approaching the golden curve via principal resonances with period $q_{n}\left(D \sim 1 / q_{n}^{\alpha}\right)$. The universal self-similar structure of diffusion between principal resonances is demonstrated and it is shown that resonances of other type play also an important role.
\end{abstract}

PACS numbers: 05.45.+b

During last years intensive investigations have allowed to understand the structure of critical invariant curves at chaos border in Hamiltonian dynamical systems with divided phase space [1,2]. Usually the analysis is carried out for two dimensional (2d) area preserving maps, the paradigm being Chirikov standard map [3]. The critical invariant curve is characterized by the rotation number and its continued fraction expansion. It has been shown that the small scale structure near invariant curves with the same tail in this expansion is universal for all smooth Hamiltonians with two degrees of freedom and for $2 \mathrm{~d}$ maps [1,2,4. Among all invariant curves better studied are those with golden rotation number $r_{g}=(\sqrt{5}-1) / 2$, whose expansion is a series of 1's. This $r_{g}$ is the most irrational number and therefore it is believed that invariant curves with golden tails are locally the most robust ones. The structure of the critical golden curve has been studied by means of renormalization group approach and it has been shown that the phase space structure is selfsimilar and universal on small scales.

Different scaling exponents have been found in this critical regime and they were successfully used to determine the diffusion rate through the destroyed invariant curve called cantorus [3,5. However, no flux passes through the golden curve at the critical value of perturbation parameter $K=K_{g}$. In this case the trajectory has only local diffusion rate $D$, which depends on its distance $\Delta r_{n}$ to the golden curve. This diffusion characterizes the motion of a particle in the vicinity of invariant curve $r_{g}$ at different levels $n$ of convergents $r_{n}=p_{n} / q_{n}$ of the continued fraction expansion of $r_{g}$ $\left(r_{1}=1 / 1, r_{2}=2 / 3, r_{3}=3 / 5 \ldots\right)$. One can expect a power law dependence of $D$ on the resonant approximant $q_{n}$, namely $D \sim q_{n}{ }^{-\alpha}$. Chirikov gave a simple argument for $\alpha=5$ [6]. According to him $D \sim\left(\Delta r_{n}\right)^{2} / t_{n}$, where $\Delta r_{n}=\left|r_{g}-r_{n}\right| \sim q_{n}^{-2}$ and $t_{n}$ is the typical inverse frequency $\Omega_{n}$ of small oscillations around principal resonance $q_{n}$. Then, $\Omega_{n} \sim q_{n} \Delta \omega_{n}$, where $\Delta \omega_{n}$ is the width of resonance $q_{n}$ [3]. In the critical case Chirikov overlap criterion [3] implies $\Delta \omega_{n} \sim \Delta r_{n}$, which gives [6]

$$
D \approx A D_{0} / q_{n}^{5} \sim\left(\Delta r_{n}\right)^{5 / 2} \sim\left(\delta y_{n}\right)^{\nu}
$$

where $D_{0}=K^{2} /\left(8 \pi^{2}\right)$ is the quasilinear diffusion rate and $\delta y_{n}=\left|y_{n}-y_{g}\right|$ is the distance of unstable periodic orbit $y_{n}$ with rotation number $r_{n}$ to the golden curve $y_{g}$ along the symmetry line. The exponent $\nu=2.14699 \ldots$ can be determined from the exponent $\sigma$ for $\delta y_{n} \sim 1 / q_{n}^{\sigma}$ found in [2] $(\nu=5 / \sigma)$.

The fast decay of diffusion rate near the chaos border $r_{g}$ means that a diffusing particle will never reach the border itself. This slow diffusion gives a long sticking of trajectory around stable islands on different renormalization levels. As a result the statistics of Poincaré recurrences $P$ (integrated probability to return into a given region after a time larger than $\tau$ ) decays with $\tau$ as $P(\tau) \sim 1 / \tau^{p}$. Such kind of decay has been first observed in [7], where the average value of the exponent $p \approx 1.5$ has been found. Further investigations have shown that the power law decay of $P$ is a generic property of Hamiltonian systems with divided phase space [8,9]. However, according to numerical results the power itself is not universal, varying in the range $1<p<2$. Moreover different maps with golden chaos boundary give different $p$ values [9], which seems to be in contradiction with universal self-similar structure of phase space near the golden border. Indeed renormalization arguments give $p=3$ 10], which is in sharp contradiction with numerical results. One of the possible reasons of the above contradiction is sticking of particles near the stable islands between principal resonances $q_{n}$. However, the attempts to take into account these intermediate resonances gave $p=2$ [10], which is too large compared to the numerical results for the golden border $1<p<1.35$ [9]. Therefore the problem of Poincaré recurrences remains unsolved and more detailed investigations of the phase space structure near the golden border should be performed. One of the reasons why the properties of $P(\tau)$ are so important is that the correlation function of dynamical variables $C(\tau)$ and the probability $\mu(\tau)$ to stay in a given region for a time $t>\tau$ are related to $P(\tau)$ 


$$
C(\tau) \sim \mu(\tau) \sim \tau P(\tau) /<\tau>\sim 1 / \tau^{p-1}
$$

where $\langle\tau\rangle$ is the average return time. The above relations follow from the ergodicity of motion on the chaotic component of the phase space [7,8,9]. The decay of correlations with power $p_{c}=p-1<1$ can lead to a divergence of global diffusion rate and to strong fluctuations in divided phase space.

In this Letter we investigate the behavior of local diffusion rate $D$ near the critical golden curve. Our first aim was to verify the theoretical prediction (1) and to analyze the structure of $D$ at different renormalization levels. As a model we have chosen Chirikov standard map

$$
\bar{y}=y-K /(2 \pi) \sin (2 \pi x) \quad, \quad \bar{x}=x+\bar{y} \quad \bmod 1
$$

with perturbation parameter corresponding to critical golden curve $K=K_{g}=.97163540631 \ldots$ To measure the local diffusion rate $D=(\Delta y)^{2} / \Delta t$ we apply the efficient method used for the investigations of Arnold and modulational diffusion in Ref. [11] ( $t$ is number of iterations). This method allows to measure very small diffusion rates (down to computer noise level) with relatively small number of iterations. By this method, we compute $D$ at different resonant Fibonacci approximants $r_{n}=p_{n} / q_{n}$ of the critical curve $r_{g}$. We use from $N_{p}=10$ to $N_{p}=100$ trajectories near unstable periodic orbits of period $q_{n}$ (these points had been determined by MacKay [2]). Each trajectory is integrated for about $T=1000 \times q_{n}$ iterations. The total interval $T$ is divided into $N_{w}=10$ windows, where the average $y$ displacement was computed with the smoothing function $f=\sin ^{2 \beta}\left(\pi t N_{w} / T\right)$. Usually we take $\beta=4 ; 6$. Such smoothing allows to suppress regular oscillations by a factor $\propto\left(N_{w} / T\right)^{4 \beta+3}$ [11]. To control the accuracy of our numerical computation of $D$, we determine $D$ also near stable periodic orbits in the center of resonance $q_{n}$, where our method gives a value $D \sim 10^{-34}$, which corresponds to the level of computer round-off error in double precision.

Our results in Fig. 1 confirm the theoretical prediction (11) for the variation of $D$ over more than 20 orders of magnitude. The numerical fit gives $\alpha=4.99 \pm 0.02$ and $A=0.0066$. We attribute the fluctuations at small $q_{n}$ values to the fact that for a large number of iterations trajectories can exit from the chaotic layer corresponding to the initial $q_{n}$. This effect disappears for larger $q_{n}$ where the local diffusion rate is sufficiently small or for shorter number of iterations. Let us note that the numerical value of $A$ is surprisingly small. Our explanation for this fact is the following. According to [3] the action change after half period of rotation in the chaotic separatrix layer is quite small $\Delta y \sim 4 \lambda^{2} \exp (-\pi \lambda / 2$ ) (here $\lambda=2 \pi$ is the frequencies ratio). This gives an order of magnitude estimate for $A=D\left(q_{n}=1\right) / D_{0} \sim(\Delta y)^{2} /\left(2 D_{0}\right) \sim 0.003$ and explains its small value. A more accurate estimate of $A$ requires taking into account higher orders of perturbation in $K$. The measured diffusion rate in the chaotic component is well separated from the diffusion in the stable regions produced by numerical round-off errors (Fig.1).

The above result shows the global structure of the diffusion rate $D$ while approaching the chaos border via resonant approximants $q_{n}$. However, an interesting question concerns the behavior of $D$ in between $r_{n}=p_{n} / q_{n}$ and $r_{n+1}$. The comparison of $D$ on these scales should reflect the self-similar structure of phase-space on different renormalization levels. To check this self-similarity we measure $D$ on two symmetry lines $x=0 ; 0.5$. The symmetry line $x=0.5$ crosses the main part of chaotic layers and contains mainly unstable points, while the other line $x=0$ passes mainly through stable islands. The known structure of periodic orbits on symmetry lines of map (3) [2] allows to find the basic renormalization intervals on stable $x=0$ and unstable $x=0.5$ lines.

For the unstable line the first renormalization level interval is $\Delta y_{1}=\left|y_{1}-y_{7}\right|$ where $y_{n}$ is the $y$ value of the unstable periodic orbit with rotation number $r_{n}$ on the line $x=0.5$. The next interval $\Delta y_{2}=\left|y_{4}-y_{10}\right|$ lies on the other side of the golden curve. The $m$-th interval is $\Delta y_{m}=\left|y_{3 m-2}-y_{3 m+4}\right|$. This $m-$ series selects the subsequence of $n$-values which we will denote by $n_{m}=3 m-2$. Intervals with odd values of $m$ lie above the invariant curve $r_{g}$ and those with even $m$ lie below. The selfsimilarity of the phase space implies that the dependence of diffusion $D$ on the position inside $m$-th interval should be approximately the same as in $m+1$-th interval after $q_{n_{m}}^{5}$ rescaling. At larger $m$-values the self-similarity is expected to become better and better. To check numerically this self-similarity we compute the diffusion rate at 320 homogeneously distributed points in the intervals $\Delta y_{m}$ for $m=1, \ldots, 6$. To compare different intervals we rescale the diffusion rate defining $D_{R}=q_{n_{m+2}}^{5} D_{m}$ where $D_{m}$ is the diffusion at $m$-th level. The position inside each interval is denoted by $\Delta y_{R}=\left|\left(y-y_{3 m-2}\right)\right| / \Delta y_{m}$ for $y$ between $y_{3 m-2}$ and $y_{3 m+4}\left(0 \leq \Delta y_{R} \leq 1\right)$.

The comparison of renormalized diffusion rate $D_{R}$ on two levels $m=4$ and $m=6$ on the unstable line is shown in Fig. 2. The diffusion rate is self-similar in agreement with universal phase space structure near the golden curve. The minimal diffusion rate $D_{R}$ on levels $m=4 ; 6$ is determined by computer noise and is different on the two levels due to the different normalization factors. The rare fluctuations in the upper diffusion plateau are presumably due to exits of trajectory from initial chaotic layer. Points with high diffusion rate are in the chaotic component, while those with minimal diffusion correspond to trajectories in stable islands. The self-similarity was also observed at other $m$ levels, both when intervals were on the same side or on opposite sides of the golden curve. This self-similarity becomes better with the growth of $m$. The sharp separation between two levels of diffusion allows to determine the leading resonances in each renormalization in- 
terval. The biggest gap in the diffusion is for periodic orbit (labelled $b$ in Fig. 2) with rotation number $r_{b}=\left[\{111\}_{m-1}, 1,2,1\right]$, where the triplet of 1's in curly brackets is repeated $m-1$ times. This rotation number is not from the series of principal resonances given by the continued fraction expansion of $r_{g}$ and labelled by $i$ in Fig. $2\left(r_{i}=\left[\{111\}_{m-1}, 1,1,1,1,1\right]\right)$. While resonance $r_{b}$ is significantly larger than the principal one $r_{i}$, there are also other resonances which are of comparable or smaller size than $r_{i}: r_{c}=\left[\{111\}_{m-1}, 1,2,1,1,1,1\right]$, $r_{d}=\left[\{111\}_{m-1}, 1,2,2,2,1\right], r_{e}=\left[\{111\}_{m-1}, 1,2,2,1\right]$, $r_{g}=\left[\{111\}_{m-1}, 1,1,1,3,2,1\right], r_{h}=\left[\{111\}_{m-1}, 1,1,1,-\right.$ $2,1,1,1], r_{l}=\left[\{111\}_{m-1}, 1,1,1,1,1,2,1,1\right]$. It is interesting to remark that most of these rotation numbers have continued fraction expansions containing mainly 1's and 2's, which is in qualitative agreement with a conjecture made in [9] on the basis of analysis of the SchmidtBialek fractal diagram of map (3). This fact is also in agreement with numerical results in [12].

In Fig. 2 we see also other types of resonances, namely $r_{a}=p_{a} / q_{a}=\left[\{111\}_{m-1}, 1,4,1\right]$ and $r_{f}=p_{f} / q_{f}=$ $\left[\{111\}_{m-1}, 1,1,1,3,1\right]$. However, they have a different structure than previous ones, displaying two and three intersections with unstable line $x=0.5$ giving rise to double $(a)$ and triple $(f)$ drops in diffusion. Indeed the periodic orbit inside resonance $r_{a}\left(r_{f}\right)$ has period $2 q_{a}$ $\left(3 q_{f}\right)$. These orbits are not present in the limit $K \rightarrow 0$, and correspond to a new chain of islands in the chaotic layer around resonances $r_{a}\left(r_{f}\right)$. The important consequence of the analysis of the structure of these resonances is that, in spite of the fact that renormalization group describes quite well the convergence to golden curve, we see that on each renormalization level resonances other than those of the main series of $r_{g}$, and even some which are not present at $K=0$, occupy a sizeable part of phase space. The existence of such non-standard resonances might explain the lack of universality of the exponent $p$ in (2) and the numerical value of $p$ significantly less than 2 . Indeed, very long Poincaré recurrences can be originated by sticking of orbits not only near the main resonances $r_{n}$ but also around these non-standard resonances and the chains of islands around them. The general description of the phase-space structure should take into account the presence of these resonances. An interesting question is which is the weakest cantorus on a given renormalization level which will determine the transition time between different renormalization levels. According to Fig.2 all drops in $D$ are associated to periodic orbits and not to invariant curves. This means that at our accuracy level there is no other invariant curve except $r_{g}$.

We have also studied the local diffusion on the stable line $x=0.0$ (Fig. 3). The size of renormalization interval $n$ is defined as $\Delta y_{n}=\left|y_{n}-y_{n+2}\right|$ and the variable $\Delta y_{R}=\left|\left(y-y_{n}\right)\right| / \Delta y_{n}$. The structure of diffusion is also self-similar. As expected, the main part of the renormalization interval is occupied by stable islands.
The largest resonances with rotation number $\rho_{a}=\left[\{1\}_{n}\right]$ and $\rho_{i}=\left[\{1\}_{n}, 1,1\right]$ correspond to the main series of $r_{g}$. There are also other resonances: $\rho_{c}=\left[\{1\}_{n}, 3,1\right]$, $\rho_{d}=\left[\{1\}_{n}, 3,1,1\right], \rho_{e}=\left[\{1\}_{n}, 2,1\right], \rho_{f}=\left[\{1\}_{n}, 2,1,1,1\right]$, $\rho_{g}=\left[\{1\}_{n}, 2,1,1\right], \rho_{h}=\left[\{1\}_{n}, 2,2,1\right]$. Again the biggest resonances have only 1's and 2's in the continued fraction. Some of the resonances seen on $x=0.0$ are also observed on $x=0.5$ and one can easily establish the correspondence. The resonance $\rho_{b}$ has the same rotation number as $\rho_{a}$, but it corresponds to a different orbit. The period of this orbit is 9 times larger than that of $\rho_{a}$ and the rotation number around main islands of $\rho_{a}$ is $1 / 9$, that is not in the golden mean sequence. This orbit is located in the center of stable islands which are embedded in the chaotic layer around principal resonances. This orbit also does not exist at $K=0$. Generally, the analysis performed on the two symmetry lines confirms the self-similar structure of diffusion and demonstrates the important role of non-standard resonances.

The above analysis allows to understand some important properties of local diffusion rate $D$ near the critical invariant curve $r_{g}$. The self-similar structure of $D$ shows also the importance of non-standard resonances, different from principal approximants $r_{n}=p_{n} / q_{n}$ of $r_{g}$. These non-standard resonances are also self-similar at different renormalization levels. However, their sizes in phase space are comparable or sometimes even larger than those of principal resonances. Therefore trajectories can be trapped for a long time around these non-standard resonances and diffuse very slowly to internal chaos boundaries surrounding islands of these resonances. Since the sizes of non-standard and principal resonances are comparable, the contribution of internal chaos boundaries to Poincaré recurrences may be relevant, and thus the decay of $P(\tau)$ may be non universal. The determination of the asymptotic behavior of $P(\tau)$ requires also a better understanding of transition rates between different renormalization levels, which are not directly related to local diffusion and should be studied in more detail.

One of us (S.R.) gratefully acknowledges the hospitality and financial support of the Laboratoire de Physique Quantique, URA 505, CNRS at Université Paul Sabatier, Toulouse, France. This work is also part of European contract \# ERBCHRXCT940460.

(a) Also: Dipartimento di Energetica, Università di Firenze, Via S. Marta, 3, 50139 Firenze, Italy and INFN, Sezione di Firenze, e-mail ruffo@vaxfi.fi.infn.it.

(b) Also: Budker Institute of Nuclear Physics, 630090 Novosibirsk, Russia.

[1] J.M. Greene, J. Math. Phys. 20, 1183 (1979). 
[2] R.S. MacKay, Physica D 7, 283 (1983).

[3] B.V. Chirikov, Phys. Rep. 52, 263 (1979).

[4] D.F. Escande, Phys. Rep. 121, 165 (1985).

[5] R.S. MacKay, J.D. Meiss and I.C. Percival, Physica D 13, 55 (1984).

[6] B.V. Chirikov, Lecture Notes in Physics 179, 29 (1983).

[7] B.V. Chirikov and D.L. Shepelyansky, Preprint INP 8169, Novosibirsk 1981 (translation Preprint PPPL-TRANS133, Plasma Phys. Lab., Princeton Univ, Princeton, New Jersey, 1983); Proc. IX Int. Conf. on Nonlinear Oscillations (Kiev 1981), Naukova Dumka 2, 420 (1984); Phys. Rev. Lett. 61, 1039 (1988).

[8] C.F.F. Karney, Physica D 8, 360 (1983).

[9] B.V. Chirikov and D.L. Shepelyansky, Physica D 13, 395 (1984); in Renormalization group, Ed. P. Exner, World Sci. Publ., Singapore, 221 (1988).

[10] J.D. Meiss and E. Ott, Physica D 20, 387 (1986).

[11] B.V.Chirikov, M.A.Lieberman, D.L.Shepelyansky and F.Vivaldi, Physica D 14, 289 (1985).

[12] J.M. Greene, R.S. MacKay and J. Stark, Physica D 21, 267 (1986)

FIG. 1. Local diffusion rate $D$ near principal resonance convergents $r_{n}=p_{n} / q_{n}$ to $r_{g}=\left(5^{1 / 2}-1\right) / 2, D_{0}=K^{2} /\left(8 \pi^{2}\right)$; chaotic component near unstable points with a) $T=10^{3} q_{n}$, $\left.N_{w}=10, N_{p}=10(\times) ; b\right) T=10^{4} q_{n}, N_{w}=100, N_{p}=100$ (O); c)regular component near stable points with $T=10^{3} q_{n}$, $N_{w}=10, N_{p}=10$ (triangles). The straight line shows the theoretical slope $\alpha=5$.

FIG. 2. Renormalized diffusion rate $D_{R} / D_{0}$ vs. rescaled position $\Delta y_{R}$ in the $m$-th level interval of renormalization scheme on the unstable symmetry line $x=0.5 ; m=4$ (full curve) and $m=6$ (dashed line with crosses). The letters indicate the drops of diffusion $D_{R}$ (see text). Other parameters are as in Fig. 1 a).

FIG. 3. Renormalized diffusion rate $D_{R} / D_{0}$ vs. rescaled position $\Delta y_{R}$ in the $n$-th level interval of renormalization scheme on the stable symmetry line $x=0.0 ; n=8$ (full curve) and $n=10$ (dashed line with crosses). The letters indicate the drops of diffusion $D_{R}$ (see text); $T=2 \times 10^{4} q_{n}$, $N_{w}=10, N_{p}=10$. 


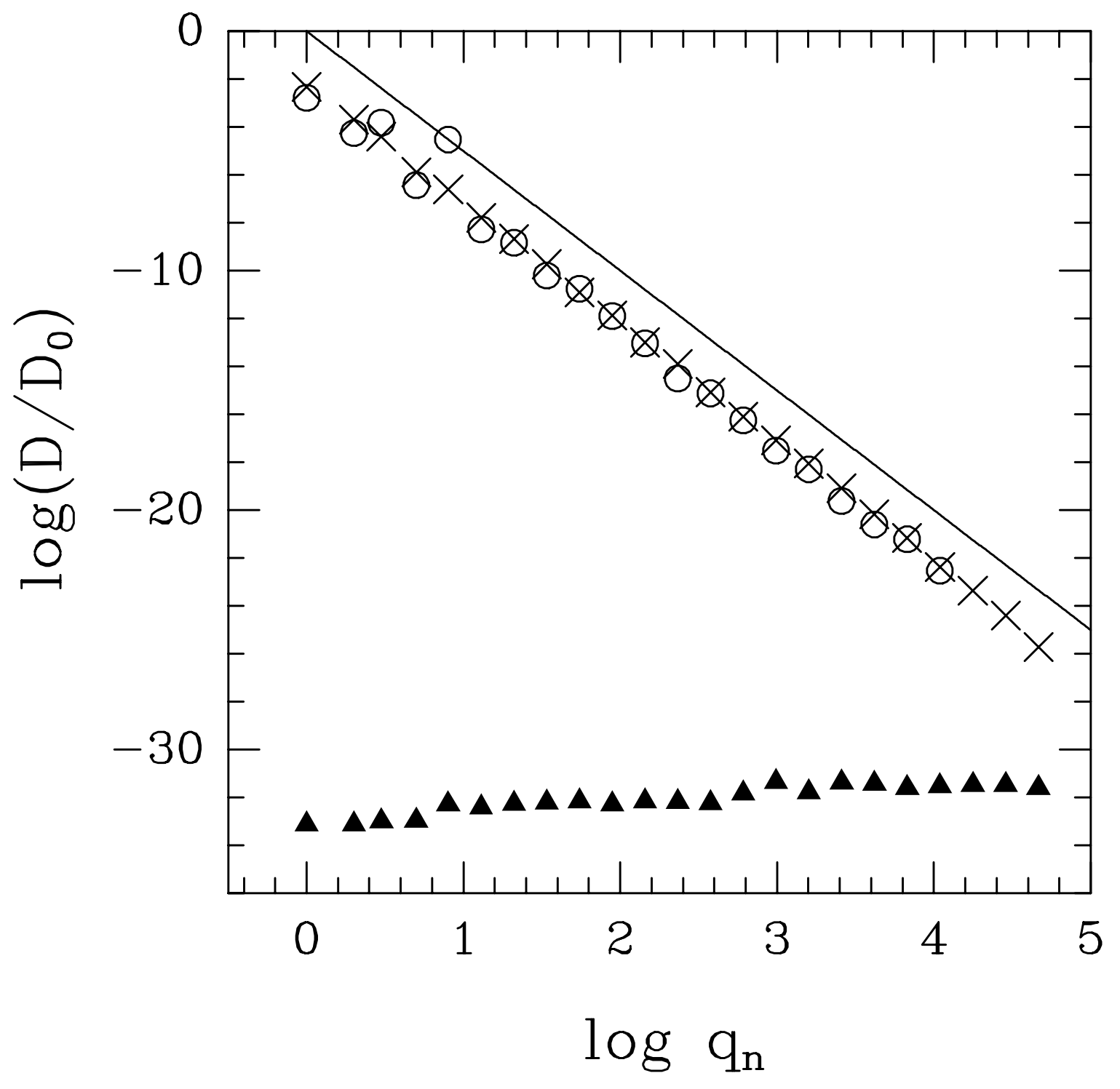




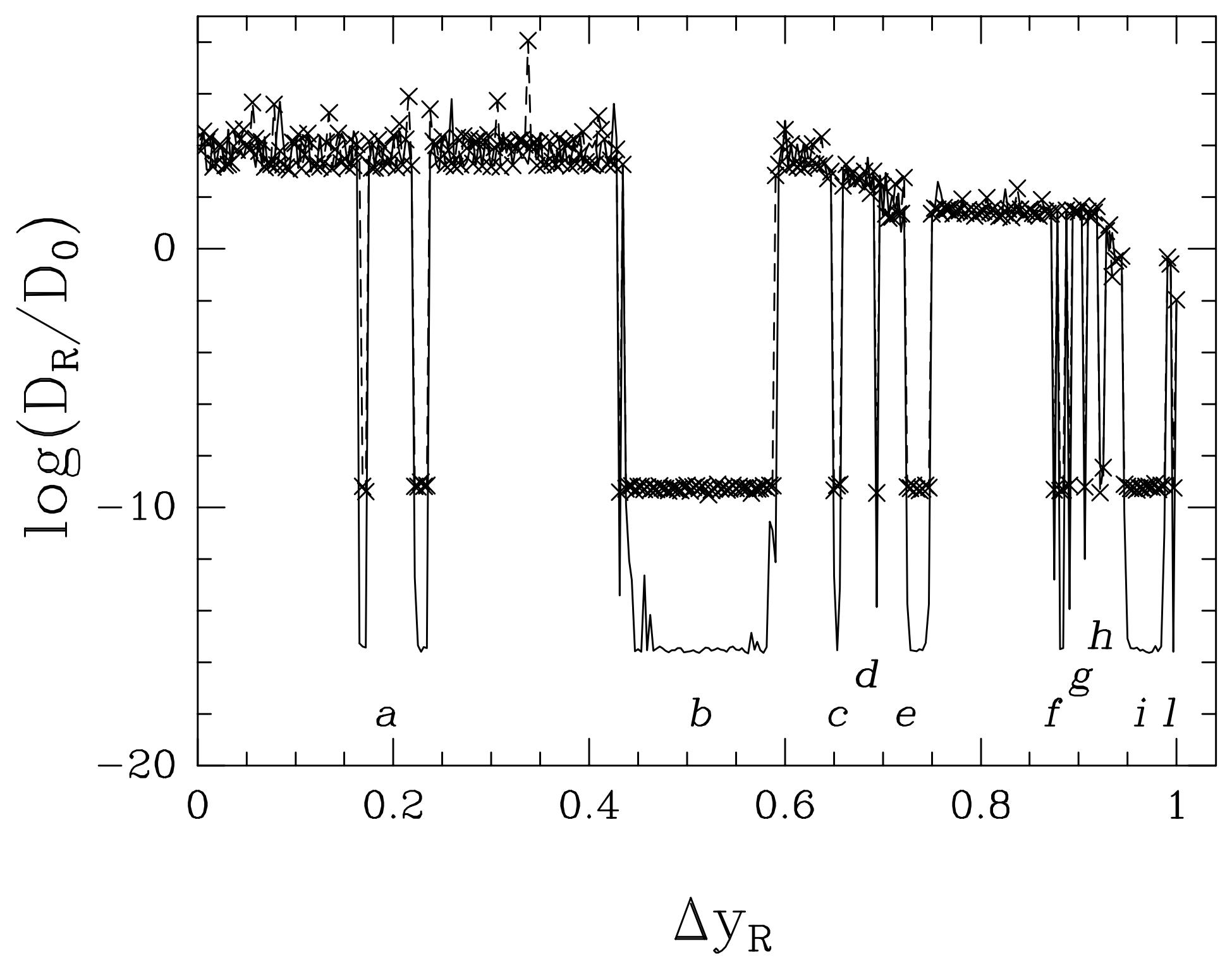




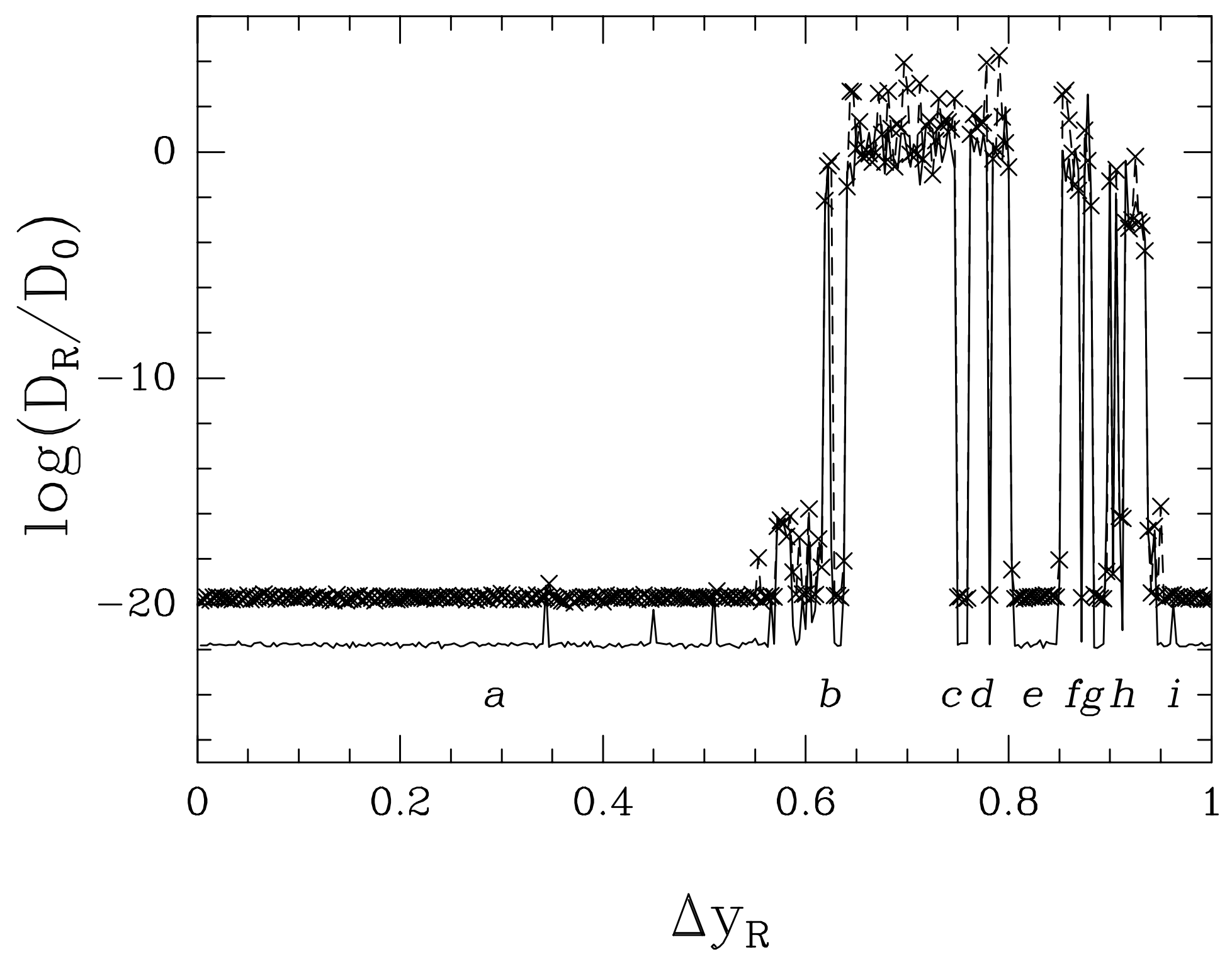

